OPEN

SUBJECT AREAS:

POLYMERS AND SOFT

MATERIALS

MATERIALS PHYSICS

CHEMICAL PHYSICS

MINERALOGY

Received

1 June 2012

Accepted

15 August 2012

Published

31 August 2012

Correspondence and requests for materials should be addressed to

E.L.H. (elisabeth. hansen@ntnu.no) or J.O.F. (jon.fossum@ ntnu.no)

* Current address:

Department of Cancer

Research and

Molecular Medicine,

Norwegian University

of Science and

Technology,

Trondheim, Norway.

$\uparrow$ Current address:

Centre de Recherche

Paul Pascal, CNRS,

Bordeaux, France.

\section{Swelling transition of a clay induced by heating}

\author{
E. L. Hansen' ', H. Hemmen' ' D. M. Fonseca' *, C. Coutant ${ }^{2 \dagger}$, K. D. Knudsen ${ }^{3}$, T. S. Plivelic ${ }^{4}$, D. Bonn ${ }^{5}$ \\ \& J. O. Fossum 1,6
}

'Department of Physics, Norwegian University of Science and Technology - NTNU, Trondheim, Norway, ${ }^{2}$ UFR Structure et Propriétés de la Matière, Université de Rennes 1, Rennes, France, ${ }^{3}$ Physics Department, Institute for Energy Technology-IFE, Kjeller, Norway, ${ }^{4}$ MAX IV Laboratory, Lund University, Lund, Sweden, ${ }^{5}$ Van der Waals-Zeeman Institute, University of Amsterdam, Amsterdam, Netherlands, ${ }^{6}$ Centre for Advanced Study - CAS, Norwegian Academy of Science and Letters, Oslo, Norway.

Clays are of paramount importance for soil stability, but also in applications ranging from oil recovery to composites and hydrogels. Generically, clays are divided into two subclasses: macroscopically swelling, 'active' clays that have the capacity for taking up large amounts of water to form stable gels, and 'passive' or non-swelling clays; the former stabilize soils whereas the latter are known to lead to landslides. However, it has been unclear so far what mechanisms underlie clay swelling. Here, we report the first observation of a temperature-induced transition from a passive to an active, swelling clay. We propose a simple description of the swelling transition; while net attractive interactions are dominant at low temperatures so that the clay particles remain attached to each other in stacks, at higher temperatures it is energetically favourable for the clay to swell due to the entropy that is gained by counterions which are liberated during swelling.

nteractions with water are determinant for the mechanical stability and material properties of clays. Understanding these complex interactions is therefore important for a wide range of fields; examples include the earth sciences ${ }^{1,2}$ and modern materials research ${ }^{3,4}$. As naturally abundant minerals, clays are important components of soils ${ }^{1}$; swelling clays subjected to wetting-and-drying cycles can for instance have huge negative impacts on the stability of constructions like buildings and roads, but also provide a natural mechanism for the recovery of a soil following compaction. In oil and gas recovery, the swelling of active clays is the principal cause of shale instabilities, which can potentially lead to wellbore collapse $\mathrm{e}^{5}$. Recent years have seen a surge of renewed interest in clays as components of novel materials, such as clay-based hydrogels ${ }^{4}$, or nano-structured claypolymer composites ${ }^{3}$. In all of these areas, the interactions of clays with water are crucial, and understanding the physical driving force for clay swelling can therefore potentially help to predict and control it.

Typically, clays consist of $1 \mathrm{~nm}$ thick crystalline phyllosilicate layers with lateral dimensions ranging from around $30 \mathrm{~nm}$ into the micrometer range ${ }^{6}$. In their dry form, the layers assemble to form stack-of-card like particles. The stacks are held together by van der Waals forces, and by electrostatic forces in the case of clays with negative layer charges. The latter types of clays have positive counterions located in between the layers of a stack, leading to an attractive interaction similar to a salt bridge $\mathrm{e}^{7,8}$, and can also take up water in the interlayers. If the water uptake is large enough, the clay is termed 'active': it will show macroscopic swelling in humid environments and develops large swelling pressures when volume changes are restricted ${ }^{5}$. We consider that, when dispersed in water, active clays may show full or partial delamination; such clays are known to form stable gels at very low volume fractions'. 'Passive' clays on the other hand have limited or no water uptake, leading to very different mechanical properties.

\section{Results}

Our study is made on a Na-fluorohectorite clay. The clay particles are polydisperse, have large lateral dimensions $(\sim \mu \mathrm{m})$ and possess a high intrinsic layer charge $\left(1.2 e^{-} \text {per unit cell }\right)^{10}$ that is balanced by $\mathrm{Na}^{+}$counterions. We studied the hydration of the samples in low-salinity water by means of synchrotron x-ray scattering. At room temperature we find that the clay is passive: its hydration is very limited and gives rise to a well-defined hydration state, with a two molecules thick layer of water forming between the layers of a particle stack. During this so-called 'crystalline swelling' ${ }^{6,11}$, particle stacks are intact and interlayer distances regular enough to give rise to welldefined diffraction peaks (Fig. 1a). Our data show that Na-fluorohectorite particles exist in aqueous solution as stacks comprised of on average roughly 100 lamellae intercalating two layers of water molecules at room 

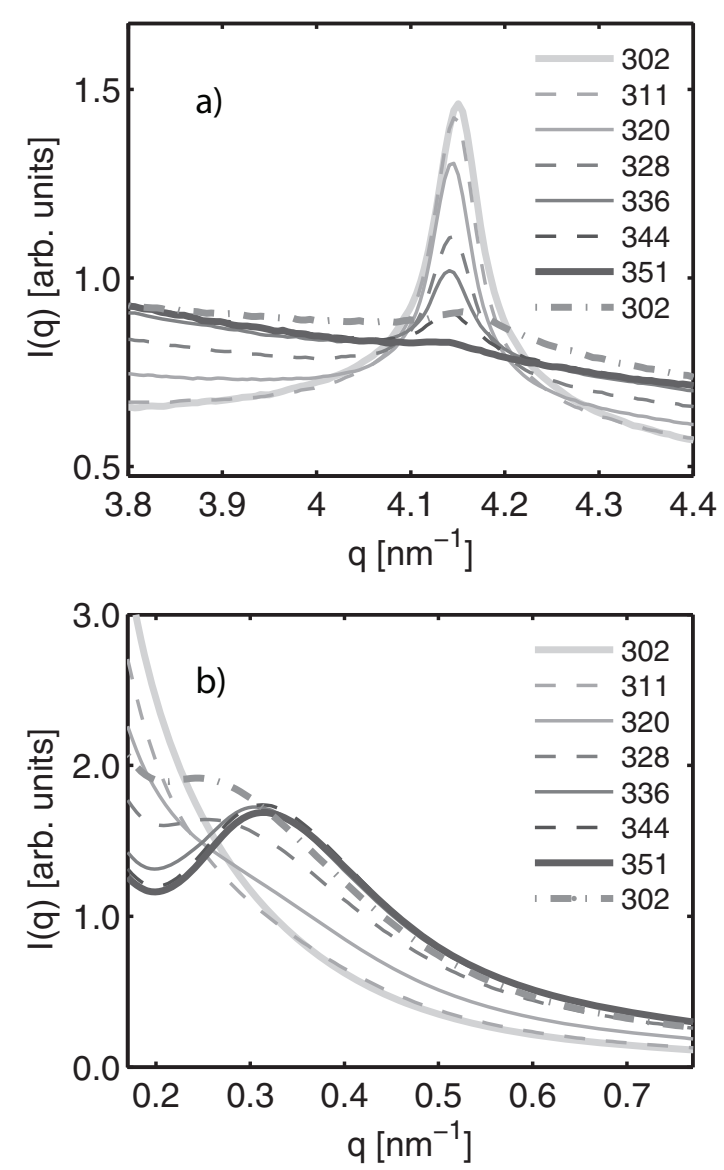

Figure $1 \mid$ Results from $x$-ray scattering. The figures show the azimuthally integrated scattered intensity as a function of the scattering vector $q$, recorded at increasing temperatures around (a) the 001 Bragg peak and (b) at small angles. Samples were prepared by dispersing $\mathrm{Na}$-fluorohectorite in $10 \mathrm{mM} \mathrm{NaCl}$ solution at a clay content of $3 \mathrm{wt} \%$. The dispersions were stored vertically for 10 days prior to measurement, allowing for some sedimentation. Diffractograms were recorded near the bottom of the capillaries where the concentration of particles was roughly $10 \mathrm{wt} \%{ }^{24}$. The scattering curves were recorded with an exposure time of 1.5 minutes starting 3 minutes after a given target temperature was achieved, with the ramp from one temperature plateau to the next lasting 10 minutes. Complimentary measurements with longer waiting times at the temperature plateaus showed no further changes in the scattering at any of the investigated temperatures, indicating that a stable state was reached quickly on the length scales investigated. The last dataset at $302 \mathrm{~K}$ was recorded post-heating after 24 hours of room temperature storage. The curves were normalized taking into account the incoming photon flux and the transmission of the sample.

temperature (see Supplementary info) ${ }^{12,13,14,15}$. The repetition distance between the clay layers is $1.51 \mathrm{~nm}$, giving rise to a Bragg peak at a scattering vector $q=4.15 \mathrm{~nm}^{-1}$ (Fig. 1a).

When the temperature is increased, large changes in the scattering are observed. The progressive disappearance of the 001 Bragg peak (Fig. 1a) shows that the stacking structure is lost. Simultaneously an intensity loss at the smallest angles and the appearance of a peak in the small-angle scattering (Fig. 1b) is consistent with the formation of a system where average interparticle correlations at large distances (approximately $2 \pi / 0.35 \mathrm{~nm}^{-1}=18 \mathrm{~nm}$ ) become progressively more predominant. Since we do not observe diffraction from other welldefined hydration states, and because the 20,13-band ${ }^{16}$ originating from lateral periodicities within clay layers remains intact (see Supplementary Fig. S2), this behaviour may only be explained by a dramatic expansion of the average interlayer distance along with the
Low temperatures

Non-swollen

particles
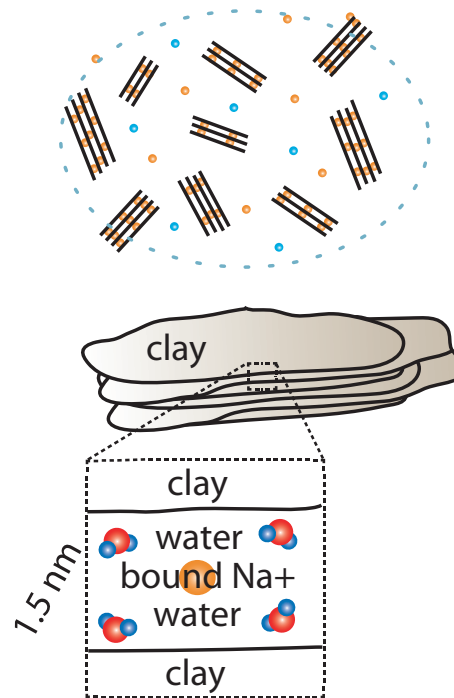

Figure $2 \mid$ Non-swelling to swelling transition. At $300 \mathrm{~K}$, non-swollen $\mathrm{Na}$-fluorohectorite particles exist as stacks intercalating two water layers. As temperature increases to $350 \mathrm{~K}$, swelling occurs as particle stacks delaminate into much smaller stacks or single unit layers and many chargebalancing interlayer cations become free to conduct.

loss of any long-range order associated with thick particle stacks. These features signify swelling and delamination. Thus the $\mathrm{Na}$ fluorohectorite particles, which are passive at low temperatures, progress towards an active, swollen state as the temperature is increased (see illustration in Fig. 2). This is to our knowledge the first observation of a non-swelling to swelling transition of a clay induced by temperature. The lack of a substantial intensity regrowth at the 001 position following cooling (Fig. 1a) shows that large particle stacks remain absent when the temperature is lowered.

This swelling transition greatly changes the material properties of the clay. The stress response $\tau(\dot{\gamma})$ of non-heated and heated samples (Fig. 3) shows that upon swelling, a yield stress emerges. To quantify the latter, measured flow curves were fitted with the HerschelBulkley model ${ }^{17}, \tau(\dot{\gamma})=\tau_{0}+k \dot{\gamma}^{n}$, with an amplitude $k$, a power law exponent $n$, and a yield stress $\tau_{0}$. The latter is observed to have increased from a negligibly small value for the non-heated system to a value of $6 \mathrm{~Pa}$ for the heated one. We attribute the appearance of a yield stress to an increase in the volume fraction of particles: when the stacked particles of an active clay delaminate into thinner stacks or into single layers, the effective excluded volume (taken as the encompassing spherical volume of the particles ${ }^{15}$ ) increases. Such an increase in the effective volume fraction is a well-known driving factor for dynamical arrest in colloidal systems.

To investigate how the temperature increase leads to swelling, we performed in-situ conductivity measurements during heating. The concentration of charge carriers as a function of temperature (Fig. 4) shows an increasing number of free (conducting) charges as the suspension is heated. This increase must be due to ions that are mobilized once the clay swells; these are most probably the $\mathrm{Na}^{+}$ counterions.

The above result allows us to postulate a simple model for the swelling. As the ions in solution are very dilute, they can be modelled as an ideal gas. We can then calculate the entropy gain of the counterions upon swelling of the clay. The Helmholtz free energy $F(T, V, N)=-k_{B} T N\left(1+\ln \left(n_{q} \frac{V}{N}\right)\right){ }^{18}$, leads to a chemical 


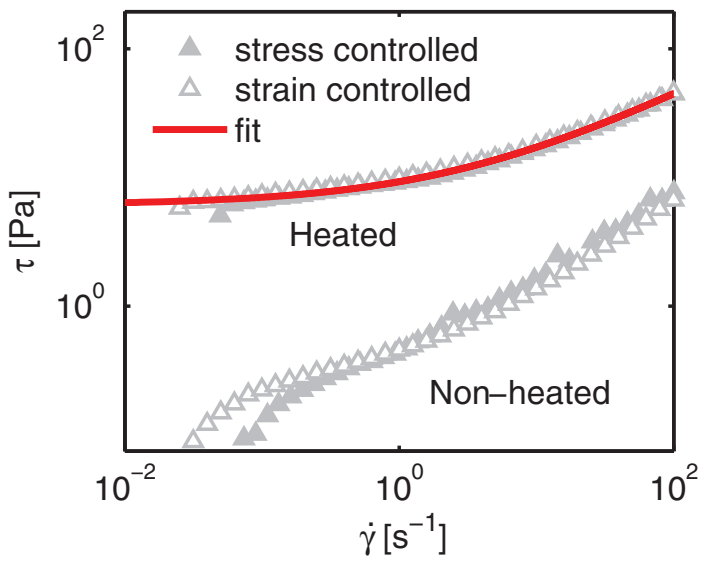

Figure 3 Rheological characterisation. Ascending flow curves for heated and non-heated samples of $10 \mathrm{wt} \% \mathrm{Na}$-fluorohectorite in $10 \mathrm{mM} \mathrm{NaCl}$ measured on a Physica MCR300 stress controlled rheometer and on a Rheometric Scientific ARES true strain controlled rheometer. Non-heated samples were prepared two days prior to measurement and kept at room temperature until the start of the experiments. Heated samples were heated to $350 \mathrm{~K}$ immediately after preparation and kept at this temperature for 15 minutes before being cooled back to room temperature and stored for two days. The reproducibility of the data obtained on the two different rheometers with different cone angles indicates that wall-slip is not significant here ${ }^{26}$. The solid line shows the Herschel-Bulkley mode ${ }^{17}$ fit. potential $\mu(T, V, N)=\left(\frac{\partial F}{\partial N}\right)_{T, V}=-k_{B} T \ln \left(n_{q} \frac{V}{N}\right)$ which is dependent on the temperature $T$ and the particle concentration $\rho=N / V$. Here $n_{q}=\left(\frac{2 \pi m k_{B} T}{h^{2}}\right)^{3 / 2}$, where $m$ is the mass of the $\mathrm{Na}^{+}$ion, $h$ is

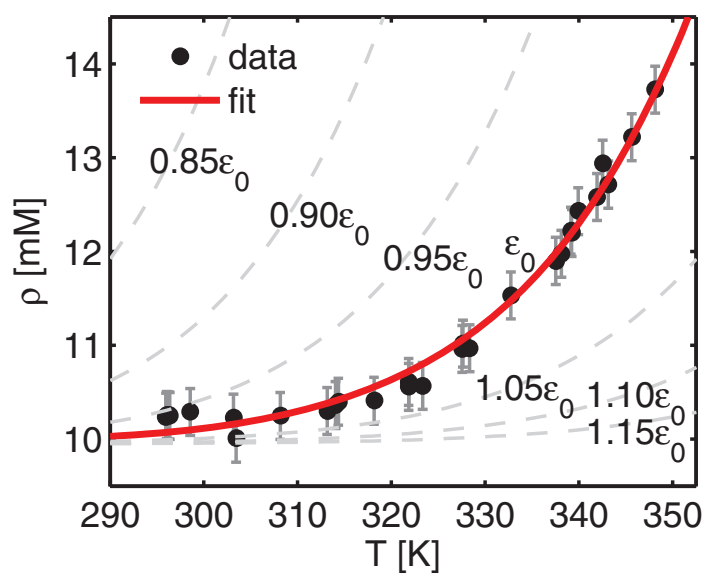

Figure $4 \mid$ Results from conductivity measurements. The figure shows the concentration of monovalent charge carriers as a function of solution temperature during heating of a $1.0 \mathrm{wt} \% \mathrm{Na}$-fluorohectorite dispersion in $10 \mathrm{mM} \mathrm{NaCl}(\bullet)$. The error bars reflect the estimated precision of the measurements, which was derived by performing the same measurements on a $\mathrm{NaCl}$ solution with a comparable and known ion concentration. The heating rate was the same as that employed in the $\mathrm{x}$-ray experiments. The temperature dependence of the conductivity due to the increased mobility as a function of temperature was corrected for. When converting from conductivity to charge carrier concentration, we have assumed all charge carriers to be monovalent and that the solution conducts the same amount of current as a $\mathrm{NaCl}$ solution would with the same number of charge carriers. The acidity was measured throughout the experiments to be between 9.50 and $9.75 \mathrm{pH}$, thus giving a negligible contribution to the conducted current. The solid line (-) is the fit as described in the text with $\varepsilon$ $=\varepsilon_{0}=-51.8 \mathrm{~kJ} / \mathrm{mol}$, and the dashed lines (--) the equilibrium concentrations projected for small deviations from $\varepsilon_{0}$.
Planck's constant and $k_{B}$ the Boltzmann constant. From the x-ray data we infer that, with the employed heating rate, swollen and nonswollen states reach equilibrium at a certain temperature $T$. Equilibrium implies that the chemical potential $\mu(T, V, N)$ of the free counterions equals the binding energy (or Gibbs free energy per particle) of the interlayer ions in the two water layer state. Now, if we assume that this energy depends only weakly on temperature for the interval considered here, $\varepsilon(T) \approx \varepsilon<0$, then from $\mu(T, V, N)=-k_{B} T \ln \left(n_{q} \frac{V}{N}\right) \approx \varepsilon$, we can deduce the equilibrium concentration $\rho$ of free $\mathrm{Na}^{+}$ions as a function of $T$ :

$$
\rho(T) \approx n_{q} \exp \left(\varepsilon / k_{B} T\right)=a T^{3 / 2} \exp \left(\varepsilon / k_{B} T\right)
$$

Here $a=\left(\frac{2 \pi m k_{B}}{h^{2}}\right)^{3 / 2}=3.44 \cdot 10^{4} \mathrm{mM} \cdot \mathrm{K}^{-3 / 2}$ for $\mathrm{Na}$. The result is in excellent agreement with the measured conductivity data (Fig. 4); from the fit we obtain a value $\varepsilon=\varepsilon_{0}=-51.8 \mathrm{~kJ} / \mathrm{mol}$.

From the nominal chemical formula for Na-fluorohectorite $\left(\mathrm{Na}_{1.2} \mathrm{Mg}_{4.8} \mathrm{Li}_{1.2} \mathrm{Si}_{8} \mathrm{O}_{20} \mathrm{~F}_{4}\right.$ per unit cell $\left.{ }^{10,19}\right)$ and the surface area of a unit cell calculated from crystallographic data, we find that our value for $\varepsilon$ is equivalent to a cohesive energy attributable to the counterions of around $-220 \mathrm{~mJ} / \mathrm{m}^{2}$. This is of the same order of magnitude as the cohesive energy of $-848 \mathrm{~mJ} / \mathrm{m}^{2}$ obtained for completely dry Na-montmorillonite through electrostatic calculations ${ }^{20}$. That our cohesive energy obtained for hydrated particles (with a layer periodicity $\approx 1.5 \mathrm{~nm}$ ) is smaller than the energy calculated for dry $\mathrm{Na}$ montmorillonite (layer periodicity $\approx 1.0 \mathrm{~nm}$ ) is expected. We also note that our swelling model is sensitive to the specific value for the binding energy (Fig. 4): it predicts that a hypothetical 15\% decrease in $|\varepsilon|$ relative to $\varepsilon=\varepsilon_{0}$ would lead to swelling already at $300 \mathrm{~K}$. On the other hand, an increase in $|\varepsilon|$ of $15 \%$ would make the swelling at $350 \mathrm{~K}$ undetectable within our experimental uncertainty. A temperature increase from 300 to $350 \mathrm{~K}$ is therefore expected to cause a passive, macroscopically non-swelling clay to become active only if its binding energy is not too different from that of Na-fluorohectorite. We note here that the layer charge of $\mathrm{Na}$-fluorohectorite is identical to the limiting layer charge $\left(1.2 e^{-}\right.$per unit cell) that is traditionally taken to separate smectites (active clays) from vermiculites and illites (passive) ${ }^{6}$.

From the nominal chemical formula for Na-fluorohectorite, we also estimate that the complete release of all $\mathrm{Na}^{+}$counterions in our $1.0 \mathrm{wt} \%$ suspension should have increased the free charge carrier concentration by around $15 \mathrm{mM}$. The fact that we observe only about one third of this increase at the highest investigated temperature could be attributed both to charges being immobilized close to the negative surfaces of the clay particles, as well as to the fact that a proportion of the particles may remain in solution as thin stacks comprising a few layers. The latter has been observed in aqueous dispersions of the active clay Laponite $\mathrm{RD}$ at room temperature ${ }^{9}$. We also observe that the swelling is not reversible by cooling alone (Fig. 1a); this may be explained by the electrostatic repulsion between layers preventing most particles from closely approaching each other once swelling has occurred.

\section{Discussion}

Our simple model (equation (1)) offers an explanation for the temperature induced non-swelling to swelling transition: the transition is driven by the increase in entropy of free counterions with temperature, and this favours swelling. Furthermore, the deduced value for the cohesive energy of $-220 \mathrm{~mJ} / \mathrm{m}^{2}$ seems reasonable. Our entropybased model resembles that developed for the swelling of polyelectrolyte gels in polar solvents: in the latter case, swelling is promoted by the increase in entropy achieved from mixing counterions with solvent within the gel $^{21}$. 
In summary, we have shown that a clay which at room temperature is passive and non-swelling, undergoes a swelling transition upon increasing the temperature. The swollen final state contains partially or fully delaminated particles plus mobile $\mathrm{Na}^{+}$ions. Such swelling could carry significance for soil mechanics ${ }^{1}$, for drilling fluids in oil wells ${ }^{5}$ and for nuclear waste storage ${ }^{22}$, which are all situations where clays in hydrous environments may encounter temperatures in the ranges studied here.

\section{Methods}

Na-fluorohectorite was prepared from synthetic Li-fluorohectorite purchased from Corning Inc., New York, through ion exchange $e^{23}$. The aqueous Na-fluorohectorite dispersions were prepared by mixing clay powder with $\mathrm{NaCl}$ salt solutions using a mechanical shaker. For the $\mathrm{x}$-ray studies, samples were prepared at $3 \mathrm{wt} \%$ solid content in $10 \mathrm{mM} \mathrm{NaCl}$, filled into $2 \mathrm{~mm}$ in diameter glass capillaries and stored vertically for 10 days prior to measurement. This aging time allows for sedimentation of large particles or aggregates and induces a vertical concentration gradient ${ }^{14,24}$. We studied the region near the bottom of the capillaries where the concentration of particles is roughly $10 \mathrm{wt} \%{ }^{24}$. The $\mathrm{x}$-ray studies were conducted at beamline I711 at MAX-lab ${ }^{25}$, and at the SNBL beamline at ESRF. Diffractograms were recorded with a counting time of 1.5 minutes starting 3 minutes after a given target temperature was reached, with the ramp of approximately $10 \mathrm{~K}$ from one temperature plateau to another lasting

10 minutes.

For the rheological study, samples were prepared at $10 \mathrm{wt} \%$ solid content in $10 \mathrm{mM} \mathrm{NaCl}$. Non-heated samples were prepared two days prior to measurement and kept at room temperature until the start of the experiments. Heated samples were heated to $350 \mathrm{~K}$ immediately after preparation and kept at this temperature for 15 minutes before being cooled back to room temperature and stored for two days. Rheological characterization was performed on a Physica MCR 300 stress controlled rheometer and on a Rheometric Scientific ARES true strain controlled rheometer. The MRC 300 featured a cone diameter of $25 \mathrm{~mm}$, a cone angle of $1.0^{\circ}$ and a $0.050 \mathrm{~mm}$ gap, and the ARES rheometer a cone diameter of $50 \mathrm{~mm}$, a cone angle of $0.040 \mathrm{rad}\left(2.3^{\circ}\right)$ and a $0.050 \mathrm{~mm}$ gap. Both rheometers were equipped with solvent traps.

The conductivity data were obtained by measuring in-situ the solution conductivity as a function of sample temperature during heating of a $1.0 \mathrm{wt} \% \mathrm{Na}-$ fluorohectorite dispersion in $10 \mathrm{mM} \mathrm{NaCl}$. The heating rate was the same as that employed in the $\mathrm{x}$-ray experiments. The temperature dependence of the conductivity due to the increased mobility as a function of temperature was corrected for. Conversion from conductivity to charge carrier concentration was performed with the assumption of all charge carriers being monovalent $\mathrm{Na}^{+}$ions.

1. Bronick, C. J. \& Lal, R. Soil structure and management: A review. Geoderma 124 , 3-22 (2005).

2. Khaldoun, A. et al. Quick Clay and Landslides of Clayey Soils. Phys. Rev. Lett. 103 (2009).

3. Bitinis, N., Hernandez, M., Verdejo, R., Kenny, J. M. \& Lopez-Manchado, M. A. Recent Advances in Clay/Polymer Nanocomposites. Advanced Materials 23, 5229-5236 (2011).

4. Wang, Q. et al. High-water-content mouldable hydrogels by mixing clay and a dendritic molecular binder. Nature (London, U.K.) 463, 339-343 (2010).

5. Anderson, R. L. et al. Clay swelling - A challenge in the oilfield. Earth-Science Reviews 98, 201-216 (2010).

6. Meunier, A. Clays. (Springer, 2005)

7. Bosshard, H. R., Marti, D. N. \& Jelesarov, I. Protein stabilization by salt bridges: concepts, experimental approaches and clarification of some misunderstandings. J. Mol. Recognit. 17, 1-16 (2004).

8. Franchi, M., Ferris, J. P. \& Gallori, E. Cations as mediators of the adsorption of nucleic acids on clay surfaces in prebiotic environments. Origins of Life and Evolution of the Biosphere 33, 1-16 (2003).

9. Ruzicka, B. et al. Competing interactions in arrested states of colloidal clays. Phys. Rev. Lett. 104, 085701 (2010).

10. DiMasi, E., Fossum, J. O., Gog, T. \& Venkataraman, C. Orientational order in gravity dispersed clay colloids: A synchrotron $x$-ray scattering study of Na-fluorohectorite suspensions. Phys. Rev. E 64, 061704 (2001).
11. Laird, D. A. Model for crystalline swelling of 2:1 phyllosilicates. Clays Clay Miner 44, 553-559 (1996).

12. da Silva, G. J., Fossum, J. O., DiMasi, E. \& Maloy, K. J. Hydration transitions in a nanolayered synthetic silicate: A synchrotron x-ray scattering study. Phys. Rev. B 67, 094114 (2003).

13. da Silva, G. J., Fossum, J. O., DiMasi, E., Maloy, K. J. \& Lutnæs, S. B. Synchrotron $\mathrm{x}$-ray scattering studies of water intercalation in a layered synthetic silicate. Phys. Rev. E 66, 011303 (2002).

14. Hemmen, H. et al. The Isotropic-Nematic Interface in Suspensions of Na-Fluorohectorite Synthetic Clay. Langmuir 25, 12507-12515 (2009).

15. Fossum, J. O. et al. Observations of orientational ordering in aqueous suspensions of a nano-layered silicate. Energy 30, 873-883 (2005).

16. Moore, D. M. \& Reynolds, R. C. X-ray diffraction and the identification and analysis of clay minerals. 2nd edn, (Oxford University Press, 1997).

17. Herschel, W. \& Bulkley, R. Konsistenzmessungen von Gummi-Benzollösungen. Colloid \& Polymer Science 39, 291-300 (1926).

18. Greiner, W., Neise, L. \& Stöcker, H. Thermodynamics and statistical mechanics. (Springer-Verlag, 1995).

19. Brigatti, M. F., Galan, E. \& Theng, B. K. G. in Developments in Clay Science Vol. Volume 1 eds F. Bergaya, B. K. G. Theng \& G. Lagaly, 19-86 (Elsevier, 2006)

20. Douillard, J. M., Salles, F., Devautour-Vinot, S., Manteghetti, A. \& Henry, M. Study of the surface energy of montmorillonite using PACHA formalism. J. Colloid Interface Sci. 306, 175-182 (2007).

21. Colby, R. H. Polyelectrolyte gels: Ionic partners split up. Nat. Mater. 6, 401-402 (2007).

22. Cuadros, J. Clay as sealing material in nuclear waste repositories. Geology Today 24, 99-103 (2008).

23. Lovoll, G. et al. Dynamics of water intercalation fronts in a nano-layered synthetic silicate: A synchrotron X-ray scattering study. Physica B (Amsterdam, Neth.) 370, 90-98 (2005).

24. Fonseca, D. M., Meheust, Y., Fossum, J. O., Knudsen, K. D. \& Parmar, K. P. S. Phase diagram of polydisperse Na-fluorohectorite-water suspensions: A synchrotron small-angle x-ray scattering study. Phys. Rev. E 79, 021402 (2009).

25. Cerenius, Y. et al. The crystallography beamline I711 at MAX II. J. Synchrotron Radiat. 7, 203-208 (2000).

26. Paredes, J., Shahidzadeh-Bonn, N. \& Bonn, D. Shear banding in thixotropic and normal emulsions. Journal of Physics-Condensed Matter 23 (2011).

\section{Acknowledgements}

The authors acknowledge beamline I711 at MAX-lab, and the SNBL beamline at the ESRF for the beamtime provided. The work received support from the Norwegian University of Science and Technology (NTNU), from the Research Council of Norway through the FRINAT and SYNKNOYT programs, and from MAX-lab through the ELISA access project. DB would like to thank Peter Bolhuis for a very helpful discussion.

\section{Author contributions}

E. L. Hansen, H. Hemmen, D. M. Fonseca, C. Coutant, K. D. Knudsen, T. S. Plivelic and J. O. Fossum participated in the synchrotron experiments. E. L. Hansen and D. Bonn did the rheological characterization and E. L. Hansen the conductivity measurements. The model for the swelling was proposed by D. Bonn. The main data analysis and writing of the article were done by E. L. Hansen, and by H. Hemmen, D. Bonn and J. O. Fossum. All authors participated in reviewing the manuscript.

\section{Additional information}

Supplementary information accompanies this paper at http://www.nature.com/ scientificreports

Competing financial interests: The authors declare no competing financial interests.

License: This work is licensed under a Creative Commons

Attribution-NonCommercial-NoDerivative Works 3.0 Unported License. To view a copy of this license, visit http://creativecommons.org/licenses/by-nc-nd/3.0/

How to cite this article: Hansen, E.L. et al. Swelling transition of a clay induced by heating Sci. Rep. 2, 618; DOI:10.1038/srep00618 (2012). 